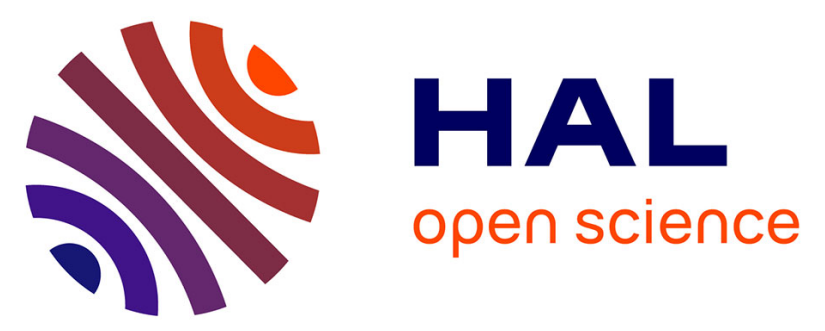

\title{
The Spatial and Temporal Harmonic Balance Method for Obtaining Periodic Responses of a Nonlinear Partial Differential Equation With a Linear Complex Boundary Condition
}

\author{
Xuefeng Wang, Weidong Zhu
}

\section{To cite this version:}

Xuefeng Wang, Weidong Zhu. The Spatial and Temporal Harmonic Balance Method for Obtaining Periodic Responses of a Nonlinear Partial Differential Equation With a Linear Complex Boundary Condition. ASME 2017 International Design Engineering Technical Conferences and Computers and Information in Engineering Conference, Aug 2017, Cleveland, OH, United States. 10.1115/DETC201767792. hal-01693118

\author{
HAL Id: hal-01693118 \\ https://hal.science/hal-01693118
}

Submitted on 25 Jan 2018

HAL is a multi-disciplinary open access archive for the deposit and dissemination of scientific research documents, whether they are published or not. The documents may come from teaching and research institutions in France or abroad, or from public or private research centers.
L'archive ouverte pluridisciplinaire HAL, est destinée au dépôt et à la diffusion de documents scientifiques de niveau recherche, publiés ou non, émanant des établissements d'enseignement et de recherche français ou étrangers, des laboratoires publics ou privés. 


\section{THE SPATIAL AND TEMPORAL HARMONIC BALANCE METHOD FOR OBTAINING PERIODIC RESPONSES OF A NONLINEAR PARTIAL DIFFERENTIAL EQUATION WITH A LINEAR COMPLEX BOUNDARY CONDITION}

\author{
Xuefeng Wang * \\ Department of Mechanical Engineering \\ University of Maryland, Baltimore County \\ Baltimore, Maryland 21250 \\ Email: xuefeng1@umbc.edu
}

\author{
Weidong Zhu \\ Department of Mechanical Engineering \\ University of Maryland, Baltimore County \\ Baltimore, Maryland 21250 \\ Email: wzhu@umbc.edu
}

\section{ABSTRACT}

The spatial and temporal harmonic balance (STHB) method is used to solve the periodic solution for a nonlinear partial differential equation (PDE) demonstrated by a nonlinear string equation with a linear complex boundary condition, and stablity analysis is conducted for the periodic solutions using Hill's method. In order to avoid the integration procedure for discretizing the PDE to obtain the ordinary differential equations (ODEs), spatial and temporal harmonic balance procedures are conducted simultaneously, which can be efficiently achieved by the discrete sine transform and the fast Fourier transform. An additional coordinate associated with the generalized coordinates of the trial functions for the spatial discretization is introduced to make the solution satisfy all boundary conditions, and a relationship of the additional coordinate and the generalized coordinates is developed and used in the STHB method so that the test functions can be the same to the trial functions. Jacobian matrix of the harmonic balanced residual is obtained analyti-

\footnotetext{
*the author's current institute is Georgia Institute of Technology, Atlanta, Georgia, 30332
}

cally, which can be used in Newton method for solving the periodic response. The STHB method and Jacobian matrix make the calculation of the periodic solution for the nonlinear string with a linear spring boundary condition efficient and easy to be implemented by computer programs. The relationship between the Jacobian matrix and the system matrix of the linearized ODEs are developed, so that one can directly obtain the Toeplitz form of the system matrix, and Hill's method can be used to analyze the stability with the eigenvalues of the Toeplitz-form system matrix without the derivation of the ODEs. The frequency curve of the periodic solutions is obtained and their stability is indicated by the method in this work.

\section{INTRODUCTION}

A nonlinear partial differential equation (PDE) can be solved by different methods, such as the perturbation methods [1], the finite element method, and Galerkin method [2]. To obtain the periodic response of the nonlinear PDE, harmonic balance method [3] and incremental harmonic balance (IHB) method [4] 
can be used for a set of ordinary differential equations (ODEs) that are obtained from the spatial discretization of the PDE by Galerkin method. However, the spatial discretization methods usually need complicated calculation to do the symbol integration. Otherwise, the numerical integration is used and it is very inefficient. A spatial and temporal harmonic balance (STHB) method is developed to solve periodic responses of a one-dimensional second-order continuous system with geometric nonlinearity and fixed-fixed boundary conditions in [5]. In the STHB method, where the spatial integration to obtain the ODEs from the PDE and the temporal harmonic balance procedure for the ODEs to solve the periodic solution are conducted simultaneously, the spatial integration is achieved by the spatial harmonic balance procedure with series of sine basis functions, which can be efficiently conducted by the fast discrete sine transform (DST) [6], and the temporal harmonic balance procedure with basis functions of Fourier series can be efficiently conducted by the fast Fourier transform (FFT) [7]. In [5], it also develops the relationship of Jacobian matrix and the system matrix of the ODEs, so that Hill's method can be used for stability analysis [8].

For the PDE with complex boundary conditions; the assumed modes method is used by employing a series of trial functions that only satisfy homogeneous boundary conditions [9]. Ren and Zhu [10] provided a substructural method of constructing trial functions, which combines the orthonormal eigenfunctions for the PDE with homogeneous boundary conditions and an additional trial function to satisfy nonhomogeneous boundary conditions.

In this work; the STHB method is used to solve the periodic response for a nonlinear string equation with a linear spring boundary condition. In order to satisfy all boundary conditions, an additional coordinate is used with generalized coordinates for the trial functions of the spatial discretization. A relationship of the additional coordinate and the generalized coordinates is developed, so that the STHB method can be used with some modification to obtain the periodic solution; and stability analysis of the solution can be conducted by Hill's method. In this method to solve the periodic response and analyze the stability, no derivation of ODEs discretizing from the PDE is required.

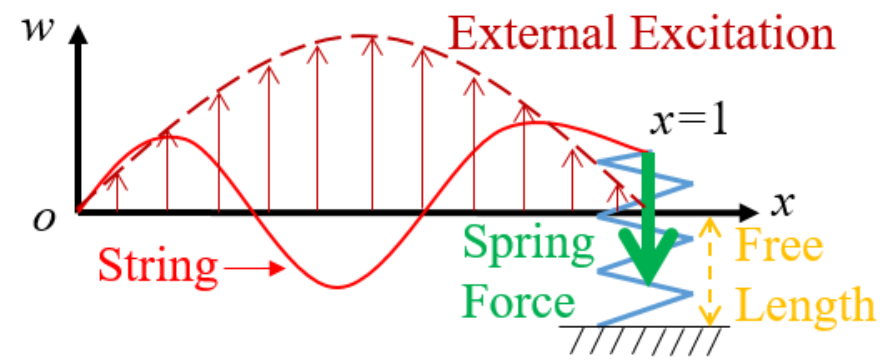

FIGURE 1. GEOMETRIC MODEL OF THE STRING WITH A SPRING BOUNDARY CONDITION

In the next section, the STHB method for solving the periodic response of the string with a linear spring boundary condition is developed. Stability analysis precedure for the obtained periodic solution is illustrated in the following. The frequency response curve of periodic solutions and their stability are shown in the last section.

\section{PERIODIC SOLUTION OF A STRING WITH A LINEAR COMPLEX BOUNDARY CONDITION}

The govening equation of a string with geometric nonlinearity in the normalized spatial and temporal coordinates, which is shown in Fig. 1, is [11]

$$
\omega^{2} w_{t t}+\omega c_{d} w_{t}-w_{x x}-k_{d} w_{x}^{2} w_{x x}=y_{0} \sin (\pi x) \cos t
$$

where $x \in[0,1]$ is the spatial coordinate, $w(x, t)$ is the transverse displacement of the string; $y_{0} \sin (\pi x) \cos t$ is the external excitation, $\omega$ is the angular excitation frequency before normalization, $c_{d}$ is the damping coefficient, and $k_{d}$ is the nonlinear stiffness coefficient. The boundary condition of the string at the fixed boundary $x=0$ is

$$
w(0,1)=0
$$


and that at the spring boundary $x=1$ is

$$
k_{0} w(1, t)+w_{x}(1, t)=0
$$

where $k_{0}$ is the stiffness of the spring. A set of trial functions of $w(x, t)$ can be

$$
\mathbf{H}_{s}=\left\{\eta_{1}(x) \cdots \eta_{N}(x)\right\}^{\mathrm{T}}
$$

with $\eta_{n}(x)=\sin (n \pi x)$, where $N$ is the number of trial functions, and a set of generalized coordinates for the trial functions are

$$
\mathbf{Q}=\left\{q_{1}(t) \cdots q_{N}(t)\right\}^{\mathrm{T}}
$$

The representation of $w(x, t)$ by $\mathbf{H}_{s}^{\mathrm{T}} \mathbf{Q}$ automatically fulfills the fixed boundary condition in Eq. (2), but it cannot satisfy the spring boundary in Eq. (3), since $\mathbf{H}_{s}^{\mathrm{T}} \mathbf{Q}$ evaluated at $x=1$ is always equal to zero. To introduce the motion at $x=1$ for the representation of $w(x, t)$, an additional trial function $e(x)=x$ with its generalized coordinate $q(t)$ is used. Since $e(x) q(t)$ at $x=0$ is also equal to zero, the fixed boundary condition is still satisfied. Thus, a solution of $w(x, t)$ can be represented by

$$
\begin{aligned}
w(x, t) & =\sum_{n=1}^{N} q_{n}(t) \eta_{n}(x)+e(x) q(t) \\
& =\mathbf{H}_{s}^{\mathrm{T}} \mathbf{Q}+e(x) q(t)
\end{aligned}
$$

The first and second partial derivatives of $w(x, t)$ with respective to $x$ are

$$
\begin{gathered}
w_{x}(x, t)=\left(\mathbf{G H}_{s}\right)^{\mathrm{T}} \mathbf{Q}+q \\
w_{x x}(x, t)=\left(\mathbf{G}_{2} \mathbf{H}_{s}\right)^{\mathrm{T}} \mathbf{Q}=\mathbf{H}_{s}^{\mathrm{T}} \mathbf{G}_{2} \mathbf{Q}
\end{gathered}
$$

respectively, where

$$
\mathbf{G H}_{s}=\{\pi \cos (\pi x) \cdots N \pi \cos (N \pi x)\}^{\mathrm{T}}
$$

with $\mathbf{G}$ being the first-order differential operator of $\mathbf{H}_{s}$, and $\mathbf{G}_{2}$ is the second-order differential operator of $\mathbf{H}_{s}$, which is a diagonal matrix with diagonal elements being $\left\{-\pi^{2} \cdots-N^{2} \pi^{2}\right\}$. Note that there is no $q$ in $w_{x x}$ since $e(x)=x$. Substituting $w(x, t)$ and $w_{x}(x, t)$ in Eqs. (6) and (7) into the spring boundary condition in Eq. (3) by setting $x=1$, one has

$$
k_{0}\left(\mathbf{H}_{s}^{\mathrm{T}}(1) \mathbf{Q}+q\right)+\left(\mathbf{G H}_{s}(1)\right)^{\mathrm{T}} \mathbf{Q}+q=0
$$

and the relationship of $q$ and $\mathbf{Q}$ is

$$
q=-\frac{1}{k_{0}+1}\left(\mathbf{G H}_{s}(1)\right)^{\mathrm{T}} \mathbf{Q}
$$

with $\mathbf{H}_{s}^{\mathrm{T}}(1)$ is a zero vector. Substituting this relationship into Eqs. (6) and (7) yields

$$
w(x, t)=\left(\mathbf{H}_{s}^{\mathrm{T}}-\frac{x}{k_{0}+1}\left(\mathbf{G} \mathbf{H}_{s}(1)\right)^{\mathrm{T}}\right) \mathbf{Q}
$$

and

$$
w_{x}(x, t)=\left(\mathbf{G H}_{s}\right)^{\mathrm{T}} \mathbf{Q}-\frac{1}{k_{0}+1}\left(\mathbf{G H}_{s}(1)\right)^{\mathrm{T}} \mathbf{Q}
$$

respectively. Substituting $w, w_{x}, w_{x x}$ given by Eqs. (10), (11) and (8) into the governing equation of the string in Eq. (1) yields

$$
\begin{array}{r}
\omega^{2}\left(\mathbf{H}_{s}^{\mathrm{T}}-\frac{x}{k_{0}+1}\left(\mathbf{G H}_{s}(1)\right)^{\mathrm{T}}\right) \ddot{\mathbf{Q}} \\
+\omega c_{d}\left(\mathbf{H}_{s}^{\mathrm{T}}-\frac{x}{k_{0}+1}\left(\mathbf{G H}_{s}(1)\right)^{\mathrm{T}}\right) \dot{\mathbf{Q}}-\mathbf{H}_{s}^{\mathrm{T}} \mathbf{G}_{2} \mathbf{Q} \\
-k_{d}\left[\left(\mathbf{G H}_{s}\right)^{\mathrm{T}} \mathbf{Q}-\frac{1}{k_{0}+1}\left(\mathbf{G H}_{s}(1)\right)^{\mathrm{T}} \mathbf{Q}\right]^{2}\left(\mathbf{H}_{s}^{\mathrm{T}} \mathbf{G}_{2} \mathbf{Q}\right) \\
=\mathbf{H}_{s}^{\mathrm{T}} \mathbf{f}
\end{array}
$$


where $\mathbf{f}=\left\{y_{0} \cos t 0 \cdots 0\right\}^{\mathrm{T}}$ is a $N \times 1$ column vector. The periodic solution of $w(x, t)$ indicates that $\mathbf{Q}$ is a periodic solution, and the $n^{\text {th }}$ element of $\mathbf{Q}$ can be written by Fourier series

$$
q_{n}(t)=\sum_{m=-M}^{M} a_{n, m} \phi_{m}(t)
$$

where $M$ is the order of Fourier series, $a_{n, m}$ is a constant coefficient of $\eta_{n}(x) \phi_{m}(t)=\eta_{n}(x) \exp (\mathrm{j} m t)$ and $\mathrm{j}$ denotes the imaginary unit. By defining $\mathbf{a}_{m}=$ $\left\{a_{1 m} \cdots a_{N m}\right\}^{\mathrm{T}}$ to be the coefficient vector for $\phi_{m}(t)$, concise expressions of $\mathbf{Q}, \dot{\mathbf{Q}}$, and $\ddot{\mathbf{Q}}$ are

$$
\begin{gathered}
\mathbf{Q}=\hat{\Phi}^{\mathrm{T}} \mathbf{a} \\
\dot{\mathbf{Q}}=\hat{\Phi}^{\mathrm{T}} \hat{\mathbf{D}} \mathbf{a} \\
\ddot{\mathbf{Q}}=\hat{\Phi}^{\mathrm{T}} \hat{\mathbf{D}}^{2} \mathbf{a}
\end{gathered}
$$

respectively, where

$$
\begin{aligned}
& \mathbf{a}=\left\{\begin{array}{lll}
\mathbf{a}_{-M}^{\mathrm{T}} & \cdots & \mathbf{a}_{M}^{\mathrm{T}}
\end{array}\right\}^{\mathrm{T}} \\
& \Phi=\left\{\phi_{-M}(t) \cdots \phi_{M}(t)\right\}^{\mathrm{T}}
\end{aligned}
$$

$\hat{\Phi}^{\mathrm{T}}=\Phi^{\mathrm{T}} \otimes \mathbf{E}_{N}, \hat{\mathbf{D}}=\mathbf{D} \otimes \mathbf{E}_{N}, \mathbf{E}_{N}$ is the $N \times N$ identity matrix, the first-order differential operator of $\Phi, \mathbf{D}$, is a diagonal matrix

$$
\mathbf{D}=\left\{\begin{array}{lll}
\mathrm{j}(-M) & & \\
& \ddots & \\
& & \mathrm{j} M
\end{array}\right\}
$$

and $\otimes$ denotes Kronecker product. The mixed-product property of Kronecker product

$$
(\mathbf{A} \otimes \mathbf{B})(\mathbf{C} \otimes \mathbf{D})=(\mathbf{A C}) \otimes(\mathbf{B D})
$$

where $\mathbf{A}, \mathbf{B}, \mathbf{C}$ and $\mathbf{D}$ are matrices of such sizes that matrix products $\mathbf{A C}$ and $\mathbf{B D}$ can be formed, can be used in the derivation of $\dot{\mathbf{Q}}$ shown in Eq. (14):

$$
\begin{aligned}
\dot{\mathbf{Q}} & =\left(\dot{\Phi}^{\mathrm{T}} \otimes \mathbf{E}_{N}\right) \mathbf{a} \\
& =\left(\left(\Phi^{\mathrm{T}} \mathbf{D}\right) \otimes\left(\mathbf{E}_{N} \mathbf{E}_{N}\right)\right) \mathbf{a} \\
& =\left(\left(\Phi^{\mathrm{T}} \otimes \mathbf{E}_{N}\right)\left(\mathbf{D} \otimes \mathbf{E}_{N}\right)\right) \mathbf{a} \\
& =\hat{\Phi}^{\mathrm{T}} \hat{\mathbf{D}} \mathbf{a}
\end{aligned}
$$

Similarly, Q̈ in Eq. (15) can be derived. Substituting $\mathbf{Q}, \dot{\mathbf{Q}}$, and $\ddot{\mathbf{Q}}$ from Eqs. (13) to (15) into Eq. (12) yields the residual function

$$
\begin{array}{r}
\mathrm{R}(\mathbf{a})= \\
\omega^{2}\left(\mathbf{H}_{s}^{\mathrm{T}}-\frac{x}{k_{0}+1}\left(\mathbf{G H}_{s}(1)\right)^{\mathrm{T}}\right) \hat{\Phi}^{\mathrm{T}} \hat{\mathbf{D}}^{2} \mathbf{a} \\
+\omega c_{d}\left(\mathbf{H}_{s}^{\mathrm{T}}-\frac{x}{k_{0}+1}\left(\mathbf{G H}_{s}(1)\right)^{\mathrm{T}}\right) \hat{\Phi}^{\mathrm{T}} \hat{\mathbf{D}} \mathbf{a}-\mathbf{H}_{s}^{\mathrm{T}} \mathbf{G}_{2} \hat{\Phi}^{\mathrm{T}} \mathbf{a} \\
-k_{d}\left[\left(\left(\mathbf{G} \mathbf{H}_{s}\right)^{\mathrm{T}}-\frac{1}{k_{0}+1}\left(\mathbf{G} \mathbf{H}_{s}(1)\right)^{\mathrm{T}}\right)\left(\hat{\Phi}^{\mathrm{T}} \mathbf{a}\right)\right]^{2} \\
\left(\mathbf{H}_{s}^{\mathrm{T}} \mathbf{G}_{2} \hat{\Phi}^{\mathrm{T}} \mathbf{a}\right)-\mathbf{H}_{s}^{\mathrm{T}} \mathbf{f}
\end{array}
$$

Conducting spatial and temporal harmonic balance procedure in [5] yields the harmonic balanced residual

$$
\mathbf{R}(\mathbf{a})=\int_{0}^{2 \pi} \frac{d t}{\pi} \int_{0}^{1} d x \hat{\Psi} \mathbf{H}_{s} \mathrm{R}(\mathbf{a})
$$

where

$$
\Psi=\left\{\phi_{M}(t) \cdots \phi_{-M}(t)\right\}^{\mathrm{T}}
$$

and $\hat{\Psi}=\Psi \otimes \mathbf{E}_{N}$. The linear part and the nonlinear part of the residual function $R(\mathbf{a})$ convert to those of the harmonic balanced residual $\mathbf{R}(\mathbf{a})$. The linear part 
of $\mathrm{R}(\mathbf{a})$ is

$$
\begin{aligned}
\mathrm{L}(\mathbf{a})= & \omega^{2}\left(\mathbf{H}_{s}^{\mathrm{T}}-\frac{x}{k_{0}+1}\left(\mathbf{G H}_{s}(1)\right)^{\mathrm{T}}\right) \hat{\Phi}^{\mathrm{T}} \hat{\mathbf{D}}^{2} \mathbf{a} \\
& +\omega c_{d}\left(\mathbf{H}_{s}^{\mathrm{T}}-\frac{x}{k_{0}+1}\left(\mathbf{G H}_{s}(1)\right)^{\mathrm{T}}\right) \hat{\Phi}^{\mathrm{T}} \hat{\mathbf{D}} \mathbf{a} \\
& -\mathbf{H}_{s}^{\mathrm{T}} \mathbf{G}_{2} \hat{\Phi}^{\mathrm{T}} \mathbf{a}-\mathbf{H}_{s}^{\mathrm{T}} \mathbf{f}
\end{aligned}
$$

and the linear part of $\mathbf{R}(\mathbf{a})$ derived in Appendix A is

$$
\begin{aligned}
\mathbf{L}(\mathbf{a}) & =\int_{0}^{2 \pi} \frac{d t}{\pi} \int_{0}^{1} d x \hat{\Psi} \mathbf{H}_{s} \mathrm{~L}(\mathbf{a}) \\
& =\omega^{2} \mathbf{E}_{2 M+1} \otimes\left(\mathbf{E}_{N}-\frac{1}{k_{0}+1} \mathbf{v}\left(\mathbf{G} \mathbf{H}_{s}(1)\right)^{\mathrm{T}}\right) \hat{\mathbf{D}}^{2} \mathbf{a} \\
& +\omega c_{d} \mathbf{E}_{2 M+1} \otimes\left(\mathbf{E}_{N}-\frac{1}{k_{0}+1} \mathbf{v}\left(\mathbf{G} \mathbf{H}_{s}(1)\right)^{\mathrm{T}}\right) \hat{\mathbf{D}} \mathbf{a} \\
& -\hat{\mathbf{G}}_{2} \mathbf{a}-\hat{\mathbf{f}}
\end{aligned}
$$

where $\mathbf{E}_{2 M+1}$ is the $2 M+1 \times 2 M+1$ identity matrix, $\mathbf{v}=\int_{0}^{1} d x \mathbf{H}_{s} x, \hat{\mathbf{G}}_{2}=\mathbf{E}_{2 M+1} \otimes \mathbf{G}_{2}$, and $\hat{\mathbf{f}}=\int_{0}^{2 \pi} \frac{d t}{2 \pi} \hat{\Psi} \mathbf{f}$. The nonlinear part of $\mathrm{R}(\mathbf{a})$ is

$$
\begin{array}{r}
\mathrm{N}(\mathbf{a})= \\
k_{d}\left[\left(\left(\mathbf{G H}_{s}\right)^{\mathrm{T}}-\frac{1}{k_{0}+1}\left(\mathbf{G H}_{s}(1)\right)^{\mathrm{T}}\right)\left(\hat{\Phi}^{\mathrm{T}} \mathbf{a}\right)\right]^{2} \\
\cdot\left(\mathbf{H}_{s}^{\mathrm{T}} \mathbf{G}_{2} \hat{\Phi}^{\mathrm{T}} \mathbf{a}\right)
\end{array}
$$

and the nonlinear part of $\mathbf{R}(\mathbf{a})$ is

$$
\mathbf{N}(\mathbf{a})=\int_{0}^{2 \pi} \frac{d t}{\pi} \int_{0}^{1} d x \hat{\Psi} \mathbf{H}_{s} \mathrm{~N}(\mathbf{a})
$$

which cannot be explicitely expressed as one can do in Eq. (19). The the STHB method in [5] can be used to calculate $\mathbf{N}(\mathbf{a})$ by discretizing spatial and temporal coordinates simultaneously. Evaluating $\mathrm{N}(\mathbf{a})$ at $\left\{x_{k}=\frac{k}{N_{S}}\right\}_{k=1, \ldots, N_{s}-1}$ and $\left\{t_{i}=\frac{2 \pi i}{M_{S}}\right\}_{i=1, \ldots, M_{s}}$, where $N_{S}>N$ and $M_{S}>2 M$, yields

$$
\tilde{\mathrm{N}}=\left\{\begin{array}{ccc}
\mathrm{N}\left(x_{1}, t_{1} ; \mathbf{a}\right) & \cdots & \mathrm{N}\left(x_{1}, t_{M_{S}} ; \mathbf{a}\right) \\
\vdots & \ddots & \vdots \\
\mathrm{N}\left(x_{N_{S}-1}, t_{1} ; \mathbf{a}\right) & \cdots & \mathrm{N}\left(x_{N_{S}-1}, t_{M_{S}} ; \mathbf{a}\right)
\end{array}\right\}
$$

Taking the fast DST for each column of $\tilde{N}$, making the elements of the corresponding column be the first $N$ coefficients of the DST, taking the FFT for each row of the resultant matrix, and making the elements of the corresponding row be the coefficients of the first $M^{\text {th }}$ order Fourier series, one has the coefficient matrix $\mathbf{C}_{s}=\left\{\begin{array}{lll}\mathbf{c}_{-M} & \ldots & \mathbf{c}_{M}\end{array}\right\}$ of $\mathrm{N}(\mathbf{a})$, so that

$$
\mathrm{N}(\mathbf{a})=\mathbf{H}_{s}^{\mathrm{T}} \mathbf{C}_{s} \Phi
$$

where $\mathbf{c}_{m}$ is a $N$-dimensional column vector with $m=$ $-M, \ldots, M$. Rearranging $\mathbf{C}_{s}$ into a column yields the harmonic balanced form of $\mathrm{N}(\mathbf{a})$, i.e.,

$$
\mathbf{N}(\mathbf{a})=\left\{\begin{array}{lll}
\mathbf{c}_{-M}^{\mathrm{T}} & \cdots & \mathbf{c}_{M}^{\mathrm{T}}
\end{array}\right\}^{\mathrm{T}}
$$

since $\mathrm{N}(\mathbf{a})=\mathbf{H}_{s}^{\mathrm{T}} \hat{\Phi} \mathbf{N}(\mathbf{a})$ and

$$
\mathbf{N}(\mathbf{a})=\int_{0}^{2 \pi} \frac{d t}{\pi} \int_{0}^{1} d x \hat{\Psi} \mathbf{H}_{s} \mathbf{H}_{s}^{\mathrm{T}} \hat{\Phi} \mathbf{N}(\mathbf{a})
$$

holds. Using $\mathbf{L}(\mathbf{a})$ and $\mathbf{N}(\mathbf{a})$ given by Eqs. (19) and (21) into the harmonic balanced residual $\mathbf{R}(\mathbf{a})$ yields

$$
\mathbf{R}(\mathbf{a})=\mathbf{L}(\mathbf{a})-\mathbf{N}(\mathbf{a})
$$

Thus, calculating the periodic solution of $w(x, t)$ changes to find a so that $\mathbf{R}(\mathbf{a})$ vanishes. One can use Newton method to solve the nonlinear problem, where the Jacobian matrix $\mathbf{R}(\mathbf{a})$ with respect to a should be obtained. Jacobian matrix of $\mathbf{L}(\mathbf{a})$ can easily obtained 
with

$$
\begin{aligned}
\mathbf{J}_{l} & =\omega^{2} \mathbf{E}_{2 M+1} \otimes\left(\mathbf{E}_{N}-\frac{1}{k_{0}+1} \mathbf{v}\left(\mathbf{G H}_{s}(1)\right)^{\mathrm{T}}\right) \hat{\mathbf{D}}^{2} \\
& +\omega c_{d} \mathbf{E}_{2 M+1} \otimes\left(\mathbf{E}_{N}-\frac{1}{k_{0}+1} \mathbf{v}\left(\mathbf{G H}_{s}(1)\right)^{\mathrm{T}}\right) \hat{\mathbf{D}} \\
& -\hat{\mathbf{G}}_{2}
\end{aligned}
$$

Jacobian matrix of $\mathbf{N}(\mathbf{a})$ can be obtained by the modification for that in [5]. Let

$$
\frac{\partial \mathbf{N}}{\partial \mathbf{a}}=\int_{0}^{2 \pi} \frac{d t}{\pi} \int_{0}^{1} d x \hat{\Psi} \mathbf{H}_{s}\left(\Gamma_{1} \frac{\partial w_{x}}{\partial \mathbf{a}}+\Gamma_{2} \frac{\partial w_{x x}}{\partial \mathbf{a}}\right)
$$

where

$$
\begin{aligned}
\Gamma_{1} & =2 k_{d} w_{x} w_{x x} \\
& =\mathbf{H}_{s}^{\mathrm{T}} \hat{\Phi}^{\mathrm{T}} \beta_{1} \\
\Gamma_{2} & =k_{d} w_{x}^{2} \\
& =\mathbf{H}_{c}^{\mathrm{T}} \hat{\Phi}^{\mathrm{T}} \beta_{2}
\end{aligned}
$$

$\beta_{1}$ and $\beta_{2}$ are harmonic balanced forms of $\Gamma_{1}$ and $\Gamma_{2}$ by the STHB method as $\mathbf{N}(\mathbf{a})$ for $\mathrm{N}(\mathbf{a})$,

$$
\begin{gathered}
\mathbf{H}_{c}=\{\cos [0 \pi x] \cdots \cos [(N-1) \pi x]\}^{\mathrm{T}} \\
\frac{\partial w_{x}}{\partial \mathbf{a}}=\left(\mathbf{G H}_{s}\right)^{\mathrm{T}} \hat{\Phi}^{\mathrm{T}}-\frac{1}{k_{0}+1}\left(\mathbf{G H}_{s}(1)\right)^{\mathrm{T}} \hat{\Phi}^{\mathrm{T}}
\end{gathered}
$$

and

$$
\frac{\partial w_{x x}}{\partial \mathbf{a}}=\mathbf{H}_{s}^{\mathrm{T}} \mathbf{G}_{2} \hat{\Phi}^{\mathrm{T}}
$$

From the derivation in [5], Jacobian matrix for the nonlinear part is

$$
\begin{aligned}
\mathbf{J}_{n}=\frac{\partial \mathbf{N}}{\partial \mathbf{a}} & =\int_{0}^{2 \pi} \frac{d t}{\pi} \hat{\Psi} \hat{\Phi}^{\mathrm{T}}\left[\left(\mathbf{E}_{2 M+1} \otimes \mathbf{M}\right) \hat{\beta}_{1}\right. \\
& \left.+\left(\mathbf{E}_{2 M+1} \otimes \mathbf{M}_{2}\right) \hat{\beta}_{2} \mathbf{G}_{2}\right] \hat{\Phi}^{\mathrm{T}}
\end{aligned}
$$

where $\hat{\beta}_{1}=\beta_{1} \otimes \mathbf{E}_{N}, \hat{\beta}_{2}=\beta_{2} \otimes \mathbf{E}_{N}$,

$$
\mathbf{M}=\mathbf{M}_{1}+\int_{0}^{1} 2 d x \mathbf{H}_{s}\left(\mathbf{H}_{s}^{\mathrm{T}} \otimes\left(\mathbf{G H}_{s}(1)\right)^{\mathrm{T}} \frac{1}{k_{0}+1}\right)
$$

$$
\mathbf{M}_{2}=\int_{0}^{1} 2 d x \mathbf{H}_{s}\left(\mathbf{H}_{c}^{\mathrm{T}} \otimes \mathbf{H}_{s}^{\mathrm{T}}\right)
$$

and $\mathbf{M}_{1}$ and $\mathbf{M}_{2}$ are constant given in [5]. For the rest part in $\mathbf{M}$, one can calculated using the mixed-product property of Kronecker product in Eq. (16):

$$
\begin{array}{r}
\int_{0}^{1} 2 d x \mathbf{H}_{s}\left(\mathbf{H}_{s}^{\mathrm{T}} \otimes\left(\mathbf{G H}_{s}(1)\right)^{\mathrm{T}} \frac{1}{k_{0}+1}\right)= \\
\left.\int_{0}^{1} 2 d x\left(\mathbf{H}_{s} \mathbf{H}_{s}^{\mathrm{T}}\right) \otimes\left(\mathbf{G H}_{s}(1)\right)^{\mathrm{T}} \frac{1}{k_{0}+1}\right)= \\
\left.\mathbf{E}_{N} \otimes\left(\mathbf{G H}_{s}(1)\right)^{\mathrm{T}} \frac{1}{k_{0}+1}\right)
\end{array}
$$

Thus, one has

$$
\left.\mathbf{M}=\mathbf{M}_{1}+\mathbf{E}_{N} \otimes\left(\mathbf{G H}_{s}(1)\right)^{\mathrm{T}} \frac{1}{k_{0}+1}\right)
$$

Using the property of Toeplitz transform in [5] for Eq. (24) yields

$$
\mathbf{J}_{n}=\int_{0}^{2 \pi} \frac{\mathrm{d} t}{\pi} \hat{\Psi} \hat{\Phi}^{\mathrm{T}} \mathbf{S}_{T} \hat{\Phi}^{\mathrm{T}}=\mathbf{S}_{\mathcal{T}}
$$


where

$$
\begin{aligned}
\mathbf{S}_{T} & =\left[\left(\mathbf{E}_{2 M+1} \otimes \mathbf{M}\right) \hat{\beta}_{1}+\left(\mathbf{E}_{2 M+1} \otimes \mathbf{M}_{2}\right) \hat{\beta}_{2} \mathbf{G}_{2}\right. \\
& =\left[\begin{array}{lll}
\mathbf{S}_{-M}^{\mathrm{T}} & \cdots & \mathbf{S}_{M}^{\mathrm{T}}
\end{array}\right]^{\mathrm{T}}
\end{aligned}
$$

and

$$
\mathbf{S}_{\mathcal{T}}=\left[\begin{array}{ccccc}
\mathbf{S}_{0} & \cdots & \mathbf{S}_{-M} & \cdots & \mathbf{0} \\
\vdots & \ddots & \vdots & \ddots & \vdots \\
\mathbf{S}_{M} & \cdots & \mathbf{S}_{0} & \cdots & \mathbf{S}_{-M} \\
\vdots & \ddots & \vdots & \ddots & \vdots \\
\mathbf{0} & \cdots & \mathbf{S}_{M} & \cdots & \mathbf{S}_{0}
\end{array}\right]
$$

is a truncated Toeplitz form of $\mathbf{S}(t)=\hat{\Phi}^{\mathrm{T}} \mathbf{S}_{T}$. Then Jacobian matrix of $\mathbf{R}(\mathbf{a})$ can be

$$
\mathbf{J}=\mathbf{J}_{l}-\mathbf{S}_{\mathcal{T}}
$$

The guess of a can be updated by Newton method to search a final solution $\mathbf{a}_{s s}$ so that $\mathbf{R}\left(\mathbf{a}_{s s}\right)=\mathbf{0}$, and Tthe periodic solution of $w(x, t)$ for the PDE in Eq. (1) is

$$
\begin{aligned}
w_{s s}(x, t) & =\left(\mathbf{H}_{s}^{\mathrm{T}}-\frac{x}{k_{0}+1}\left(\mathbf{G H}_{s}(1)\right)^{\mathrm{T}}\right) \mathbf{Q}_{s s} \\
& =\left(\mathbf{H}_{s}^{\mathrm{T}}-\frac{x}{k_{0}+1}\left(\mathbf{G H}_{s}(1)\right)^{\mathrm{T}}\right) \hat{\Phi}^{\mathrm{T}} \mathbf{a}_{s s}
\end{aligned}
$$

\section{STABILITY ANALYSIS OF THE PERIODIC SOLUTION}

For stability analysis of $w_{s s}(x, t)$ for Eq. (1), one can study its associated ODEs that are obtained by using Galerkin procedure for Eq. (12), where the test functions are $\mathbf{H}_{s}$ :

$$
\begin{array}{r}
2 \int_{0}^{1} d x \mathbf{H}_{s}\left[\omega^{2}\left(\mathbf{H}_{s}^{\mathrm{T}}-\frac{x}{k_{0}+1}\left(\mathbf{G} \mathbf{H}_{s}(1)\right)^{\mathrm{T}}\right) \ddot{\mathbf{Q}}\right. \\
\left.+\omega c_{d}\left(\mathbf{H}_{s}^{\mathrm{T}}-\frac{x}{k_{0}+1}\left(\mathbf{G H}_{s}(1)\right)^{\mathrm{T}}\right) \dot{\mathbf{Q}}-\mathbf{H}_{s}^{\mathrm{T}} \mathbf{G}_{2} \mathbf{Q}\right] \\
-\mathcal{L}(\mathbf{Q})-\mathbf{f}=0
\end{array}
$$

where

$$
\begin{aligned}
\mathcal{L}(\mathbf{Q}) & =2 \int_{0}^{1} d x \mathbf{H}_{s} k_{d}\left[\left(\mathbf{G} \mathbf{H}_{s}\right)^{\mathrm{T}} \mathbf{Q}\right. \\
& \left.-\frac{1}{k_{0}+1}\left(\mathbf{G H}_{s}(1)\right)^{\mathrm{T}} \mathbf{Q}\right]^{2}\left(\mathbf{H}_{s}^{\mathrm{T}} \mathbf{G}_{2} \mathbf{Q}\right)
\end{aligned}
$$

The simplified expression of Eq. (28) is

$$
\begin{array}{r}
\omega^{2}\left(\mathbf{E}_{N}-\frac{1}{k_{0}+1} \mathbf{v}\left(\mathbf{G H}_{s}(1)\right)^{\mathrm{T}}\right) \ddot{\mathbf{Q}}= \\
-\omega c_{d}\left(\mathbf{E}_{N}-\frac{1}{k_{0}+1} \mathbf{v}\left(\mathbf{G H}_{s}(1)\right)^{\mathrm{T}}\right) \dot{\mathbf{Q}} \\
+\mathbf{G}_{2} \mathbf{Q}+\mathcal{L}(\mathbf{Q})+\mathbf{f}
\end{array}
$$

Linearization of Eq. (30) about the periodic solution $\mathbf{Q}_{s s}=\hat{\Phi}^{\mathrm{T}} \mathbf{a}_{s s}$ is

$$
\begin{array}{r}
\omega^{2}\left(\mathbf{E}_{N}-\frac{1}{k_{0}+1} \mathbf{v}\left(\mathbf{G H}_{s}(1)\right)^{\mathrm{T}}\right) \ddot{\mathbf{Q}}= \\
-\omega c_{d}\left(\mathbf{E}_{N}-\frac{1}{k_{0}+1} \mathbf{v}\left(\mathbf{G H}_{s}(1)\right)^{\mathrm{T}}\right) \dot{\mathbf{Q}} \\
+\mathbf{G}_{2} \mathbf{Q}+\frac{\partial}{\partial \mathbf{Q}}\left(\mathcal{L}\left(\mathbf{Q}_{s s}\right)\right) \mathbf{Q}
\end{array}
$$

and its state-space form is

$$
\mathbf{B} \dot{\gamma}=\mathbf{A}(t) \gamma
$$

where $\gamma=\left\{\mathbf{Q}^{\mathrm{T}}, \dot{\mathbf{Q}}^{\mathrm{T}}\right\}^{\mathrm{T}}$ is the state variable vector

$$
\mathbf{B}=\left\{\begin{array}{l}
\mathbf{E}_{N} \quad \mathbf{0}_{N} \\
\mathbf{0}_{N} \mathbf{E}_{N}-\frac{1}{k_{0}+1} \mathbf{v}\left(\mathbf{G} \mathbf{H}_{s}(1)\right)^{\mathrm{T}}
\end{array}\right\}
$$

is a constant matrix, and

$$
\mathbf{A}(t)=\left\{\begin{array}{c}
\mathbf{0}_{N} \\
\frac{1}{\omega^{2}}\left(\mathbf{G}_{2}+\frac{\partial \mathcal{L}}{\partial \mathbf{Q}}\right)-\frac{c_{d}}{\omega}\left(\mathbf{E}_{N}-\frac{1}{k_{0}+1} \mathbf{v}\left(\mathbf{G H}_{s}(1)\right)^{\mathrm{T}}\right)
\end{array}\right\}
$$

is a periodic matrix. Due to the nonlinear term $\frac{\partial \mathcal{L}}{\partial \mathbf{Q}}$ in $\mathbf{A}(t)$, one needs to do the integration in Eq. (29) by handwork, which is workable only for simple nonlinear- 
ity and needs lots of work, or by numerical integration that takes very long time. However, the discussion in [5] indicates a relationship between nonlinear terms in $\mathbf{A}(t)$ and Jacobian matrix $\mathbf{J}$, which is

$$
\frac{\partial \mathcal{L}}{\partial \mathbf{Q}}=\hat{\Phi}^{\mathrm{T}} \mathbf{S}_{T}=\mathbf{S}(t)
$$

With this relationship, stability of $w_{s s}(x, t)$ can be analyzed by Hill's method. A truncated Toeplitz form of $\mathbf{A}(t)$, denoted by $\mathbf{A}_{\mathcal{T}}$, has the same form as Eq. (26), whose diagonal block is

$$
\begin{array}{r}
\mathbf{A}_{0}=\left\{\begin{array}{c}
\mathbf{0}_{N} \\
\frac{1}{\omega^{2}} \mathbf{G}_{2}-\frac{c_{d}}{\omega}\left(\mathbf{E}_{N}-\frac{1}{k_{0}+1} \mathbf{v}\left(\mathbf{G H}_{s}(1)\right)^{\mathrm{T}}\right)
\end{array}\right\} \\
+\left\{\begin{array}{cc}
\mathbf{0}_{N} & \mathbf{0}_{N} \\
\frac{1}{\omega^{2}} \mathbf{S}_{0} & \mathbf{0}_{N}
\end{array}\right\}
\end{array}
$$

and off-diagonal blocks are

$$
\mathbf{A}_{m}=\left\{\begin{array}{cc}
\mathbf{0}_{N} & \mathbf{0}_{N} \\
\frac{1}{\omega^{2}} \mathbf{S}_{m} & \mathbf{0}_{N}
\end{array}\right\}, m \in\{-M \cdots-1,1 \cdots M\}
$$

where $\mathbf{S}_{m}$ is the coefficient matrix of $\mathbf{S}(t)$, which can be directly obtained from $\mathbf{S}_{T}$ in $\mathbf{J}$. A truncated Toeplitz form of $\mathbf{B}$ denoted by $\mathbf{B}_{\mathcal{T}}$ is a diagonal matrix with the diagonal block as $\mathbf{B}$ itself. From Floquet theory, locations of eigenvalues of $\mathbf{B}_{\mathcal{T}}^{-1} \mathbf{A}_{\mathcal{T}}$ on the complex plane are repeated in strips $(\mathrm{j}(2 k-1) \pi, \mathrm{j}(2 k+1) \pi]$ along the imaginary axis direction with the integer $k \in(-\infty,+\infty)$. Eigenvalues $\lambda=\left[\begin{array}{lll}\lambda_{1} & \cdots & \lambda_{N}\end{array}\right]$ in the fundamental strip $(-\mathrm{j} \pi, \mathrm{j} \pi]$ are used to analyze stability of Eq. (31), which indicates the local stability of $w_{s s}(x, t)$. If all values of $\exp \left(2 \pi \lambda_{n}\right)$ with $n \in\{1 \cdots N\}$ are inside the unit circle, $w_{s s}(x, t)$ is stable; otherwise, it is unstable. Furthermore, in an unstable case, if there are values of $\exp \left(2 \pi \lambda_{n}\right)$ less than -1 , there occurs a period-doubling bifurcation; if there are values of $\exp \left(2 \pi \lambda_{n}\right)$ that are complex, a Hopf bifurcation occurs.

\section{RESULTS}

In the simulation, some values of coefficients in Eq. (1) are chosen as $c_{d}=1.2, y_{0}=1.5, k_{d}=10, N=5$, $M=11, N_{s}=32$ and $M_{s}=64$. When the spring stiffness $k_{0}=10$ and $\omega=1.5$, the periodic solution $w_{s s}(x, t)$ is achieved by the method above. Responses of the generalized coordinates $\mathbf{Q}=\left\{q_{1}(t) \cdots q_{N}(t)\right\}^{\mathrm{T}}$ and the additional coordinate $q(t)$ in one period are shown in Fig. 2. Since $k_{0}$ is small, amplitude of the response $q(t)$ at the spring boundary is about one third of that of the response $q_{1}(t)$ for the $1^{\text {th }}$ order trial function $\eta_{1}(t)$. Responses of the periodic solution of $w(x, t)$ at different time points from zero to $2 \pi$ are shown in Fig. 3. Due to the spring boundary, the motion at the boundary $x=1$ is not stationary. Stability analysis from the previous section shows that the periodic solution $w_{s s}(x, t)$ is stable with the eigenvalues equal to

$\lambda=\{-0.08 \pm \mathrm{j} 0.02,-0.06 \pm \mathrm{j} 0.05,0.04 \pm \mathrm{j} 0.07,0.08 \pm \mathrm{j} 0.02\}$

When $k_{0}=50$, responses of $\mathbf{Q}$ and $q$ in Fig. 4 show that the motion at the spring boundary is much smaller than that of the string. The frequency response curve in Fig. 5 is plotted to show the relationship of the norm of $\mathbf{a}$ and frequency $\omega$, and unstable solutions are indicated as well. Stability analysis in the previous section also indicates that there is no period-doubling or Hopf bifurcations for the unstable cases. Different numbers of trial functions, $N$, and orders of Fourier series, $M$, are used to verify solutions from the STHB method, as shown in Fig. 5.

\section{CONCLUSION}

The STHB method is demonstrated to calculate the periodic responses for a nonlinear string with a linear spring boundary condition. A spectral method is used to discretize the PDE to sets of ODEs, where an additional coordinate is introduced to make the solution satisfy both the fixed and spring boundary conditions. The relationship of the additional coordinate and the generalized coordinates of basis functions for the spectral method is found, so that the test functions can be the same to the trial functions, and the 


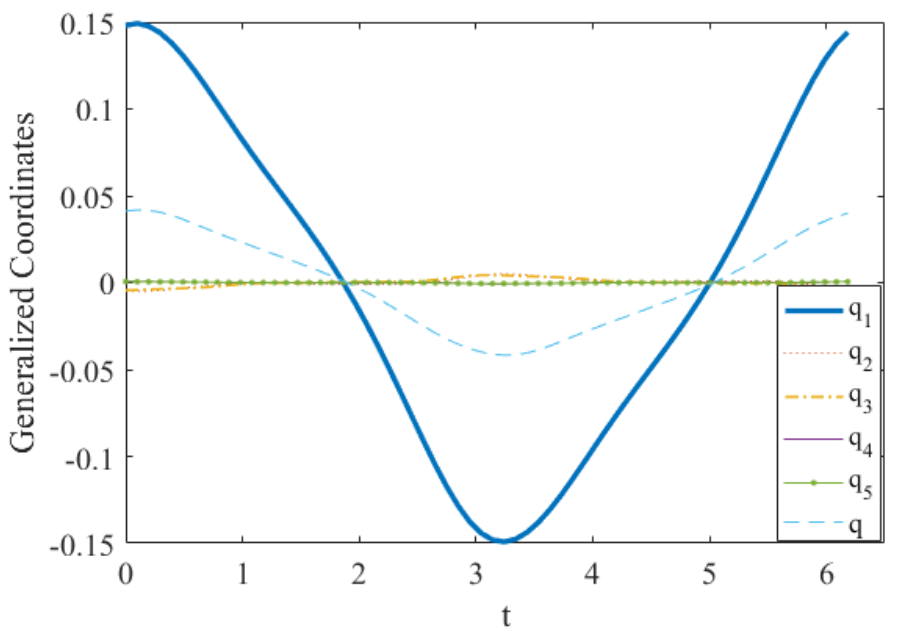

FIGURE 2. RESPONSES OF Q AND $q$ IN ONE PERIOD WITH $k_{0}=10$

STHB method can be used here to automatically and efficiently obtain the periodic solution of the nonlinear string equation with the linear spring boundary, where the derivation of the ODEs are not required. In stability analysis, the relationship of Jacobian matrix and the system matrix of the ODEs is used to obtain the Toeplitz form of the system matrix, so that the derivation of the ODEs can be also avoided, which makes this method efficient and easy to be mplemented. The frequency response curve of periodic solutions is obtained, and the stability of the solutions is indicated.

\section{ACKNOWLEDGMENT}

The authors would like to thank the technical advision from Wei Fan.

\section{REFERENCES}

[1] Nayfeh, A. H., 1998. "Reduced-order models of weakly nonlinear spatially continuous systems". Nonlinear Dynamics, 16(2), pp. 105-125.

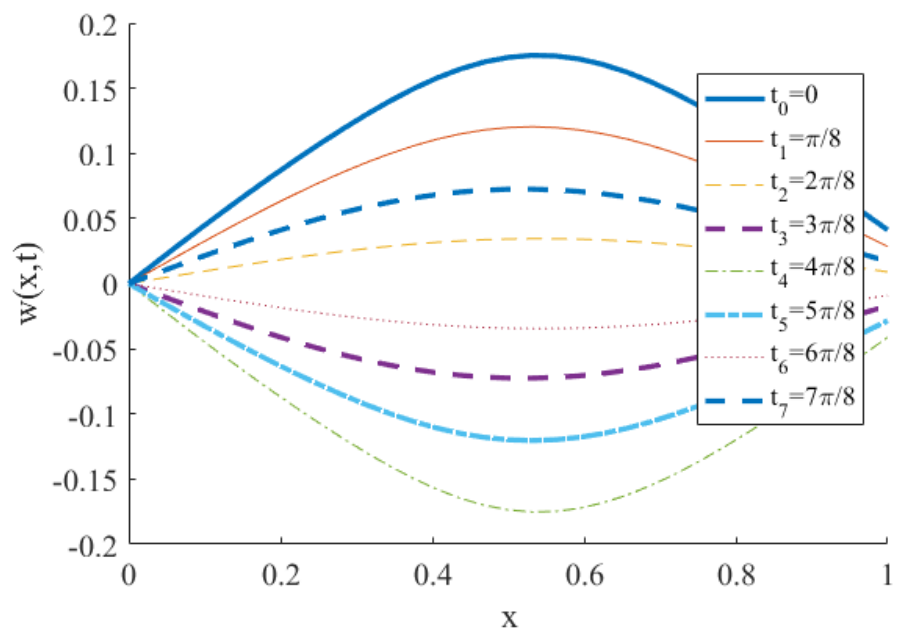

FIGURE 3. TIME RESPONSES OF $w(x, t)$ WITH $k_{0}=$ 10

[2] Meirovitch, L., 1997. Principles and techniques of vibrations, Vol. 1. Prentice Hall New Jersey.

[3] Szemplinska-Stupnicka, W., 1978. "The generalized harmonic balance method for determining the combination resonance in the parametric dynamic systems". Journal of Sound and Vibration, 58(3), pp. 347-361.

[4] Lau, S., Cheung, Y., and Wu, S., 1982. "A variable parameter incrementation method for dynamic instability of linear and nonlinear elastic systems". ASME J. Appl. Mech, 49(4), pp. 849-853.

[5] Wang, X., and Zhu, W., 2016. "A new spatial and temporal incremental harmonic balance method for obtaining steady-state responses of a one-dimensional continuous system". Journal of Applied Mechanics.

[6] Chivukula, R. K., and Reznik, Y. A., 2011. "Fast computing of discrete cosine and sine transforms of types vi and vii". In SPIE Optical Engineering+ Applications, International Society for Optics and Photonics, pp. 813505-813505.

[7] Wang, X., and Zhu, W., 2015. "A modified 


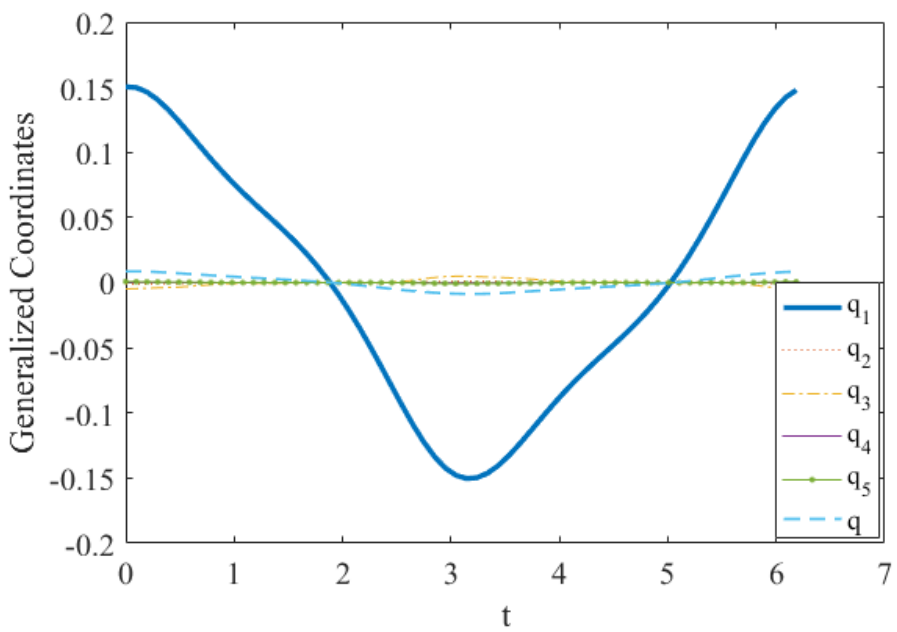

FIGURE 4. RESPONSES OF Q AND $q$ IN ONE PERIOD

WITH $k_{0}=50$

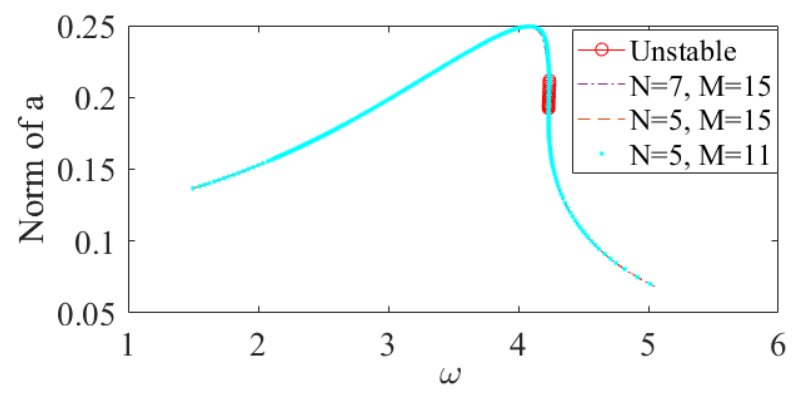

FIGURE 5. FREQUENCY-RESPONSE CURVES WITH $k_{d}=10$

incremental harmonic balance method based on the fast fourier transform and broyden's method". Nonlinear Dynamics, 81(1-2), pp. 981-989.

[8] Wereley, N. M., 1990. "Analysis and control of linear periodically time varying systems". $\mathrm{PhD}$ the- sis, Massachusetts Institute of Technology.

[9] Xu, G., and Zhu, W., 2010. "Nonlinear and timevarying dynamics of high-dimensional models of a translating beam with a stationary load subsystem". Journal of Vibration and Acoustics, 132(6), p. 061012.

[10] Ren, H., and Zhu, W., 2013. "An accurate spatial discretization and substructure method with application to moving elevator cable-car systems part ii". Journal of Vibration and Acoustics, $135(5)$, p. 051037.

[11] Mote, C., 1966. "On the nonlinear oscillation of an axially moving string". Journal of Applied Mechanics, 33(2), pp. 463-464. 